\title{
Inflammatory Myofibroblastic Tumor of Urinary Bladder
}

\author{
Ronica Baruah ${ }^{1}$, Abhijit Kalita ${ }^{1 *}$ and Phanindra Mohan Deka ${ }^{2}$ \\ 'Department of Pathology, Ekopath Metropolis Lab Services Pvt. Ltd. Guwahati, Assam, India \\ ${ }^{2}$ Department of Urology, Dispur Hospitals Pvt. Ltd. Guwahati, Assam, India
}

\begin{abstract}
Inflammatory myofibroblastic tumor (IMT) is an uncommon benign tumor of intermediate neoplastic potential, characterised by spindle cell proliferation with characteristic fibroinflammatory and pseudosarcomatous appearance. A subset of IMT with histologic atypia and/or clinical aggressiveness is also known. IMTs occur in the mesentery, omentum, retroperitoneum, pelvis, and abdominal soft tissues. However, the occurrence of IMT in urinary bladder is unusual. IMT exhibits morphologic and immunophenotypic overlap with malignant spindle cell tumors of the urinary bladder and is diagnostically challenging. In the case presented, ALK-1 and SMA immunostains helped to identify IMT. Anaplastic Lymphoma Kinase (ALK) gene translocation or ALK gene expression can further confirm IMT. Complete surgical excision with follow-up is the treatment of choice for IMT.
\end{abstract}

Keywords: Inflammatory Myofibroblastic Tumor, ALK-1 Immunostain, Spindle Cell Proliferation.

\section{Introduction}

Inflammatory myofibroblastic tumor (IMT) is a distinctive neoplasm composed of myofibroblastic and fibroblastic spindle cells accompanied by inflammatory infiltration of plasma cells, lymphocytes, and eosinophils ${ }^{[1,2]}$. IMT is also known as pseudosarcoma, atypical myofibroblastic tumor, atypical fibromyxoid tumor and pseudosarcomatous appearance. IMT is a neoplasm of intermediate biologic potential $^{[3]}$. A subset of IMT is identified with histologic atypia and clinical aggressiveness, and may be difficult to distinguish from other sarcomatous proliferations. It may affect any age group, but it is more common in children and young adults with slight female preponderance. The origin of IMT is controversial, but a recent report suggests that it is neoplastic because of aggressive behaviour, involvement of chromosome 2p23, and congenital clonality. IMTs occur in the mesentery, omentum, retroperitoneum, pelvis, and abdominal soft tissues. However, the occurrence of IMT in urinary bladder is unusual ${ }^{[1]}$. IMT exhibits morphologic and immunophenotypic overlap with malignant spindle cell tumors of the urinary bladder and diagnostic distinction from these tumors can be problematic. Both epithelial and myogenic markers can be expressed in IMT and may lead to a misdiagnosis of sarcomatoid carcinoma, leiomyosarcoma, and rhabdomyosarcoma ${ }^{[4]}$. The treatment of choice is total excision of the tumor ${ }^{[5]}$.

\section{Case Report}

A 45 year old male presented with painless hematuria, clots in urine and burning micturition for one month. No past history or family history of any specific disease was elicited. Urine microscopic examination showed plenty of red cells as the only abnormal finding. Cystoscopic examination was done which showed a large number of blood clots which were subsequently evacuated. Following evacuation of the clot, a large broad based solid tumor was found in the posterior wall of the urinary bladder for which transurethral resection of the tumor was done and biopsy sent for histopathological examination.

The tissue received consisted of multiple bits aggregating to $3 \times 3 \times 1 \mathrm{~cm}$. Sections from the formalin fixed paraffin embedded bladder mass stained with Hematoxylin and Eosin showed spindle cell proliferation with moderate nuclear atypia, occasional mitotic figures and few scattered lymphocytes. Similar spindle cells were also seen infiltrating the muscularis mucosae and involving the base of tumor (Figure 1). It presented as a malignant spindle cell tumor. However, there was absence of atypical mitotic figures and areas of necrosis, presence of delicate abundant vascular network, scattered oedematous / myxoid areas and inflammatory cells in deeper layers of the tumor. These features prompted a possible benign nature of the tumor. A provisional diagnosis of Sarcomatoid Transitional cell carcinoma was given based on the location of the tumor, invasion of muscular layer and nuclear atypia. Immunohistochemistry was recommended for final diagnosis.

Considering various possibilities, a battery of immunostains were done on the tumor section, to come to a final diagnosis. The immunostains included Ki 67, CK 7, CK 20, Uroplakin, S-100, Chromogranin, Synaptophysin, 
EMA, desmin, SMA, and ALK-1. The tumor showed a high proliferative index (high Ki 67 - Figure 2). Transitional carcinoma was ruled out following negative staining for CK20, CK7 and Uroplakin. S-100, EMA and desmin were also negative, ruling out the possibility of the tumor having neural or muscular origin. Neuroendocrine lesion was ruled out based on negativity for synaptophysin and chromogranin. However, SMA showed focal positivity and ALK-1 showed strong positivity (Figure 2).
The immunohistochemistry results were compiled and most sarcomatous lesions were ruled out. Consequently, a benign spindle cell lesion, either IMT or Post-operative Spindle Cell Nodule (PSCN) were considered in the differential diagnosis. PSCN was excluded as there was no past history of any surgical intervention or biopsy. So, a final diagnosis of IMT was given. However, translocation study of ALK gene was recommended (by cytogenetics or Fluorescent-in-situ Hybridisation) for the confirmation.

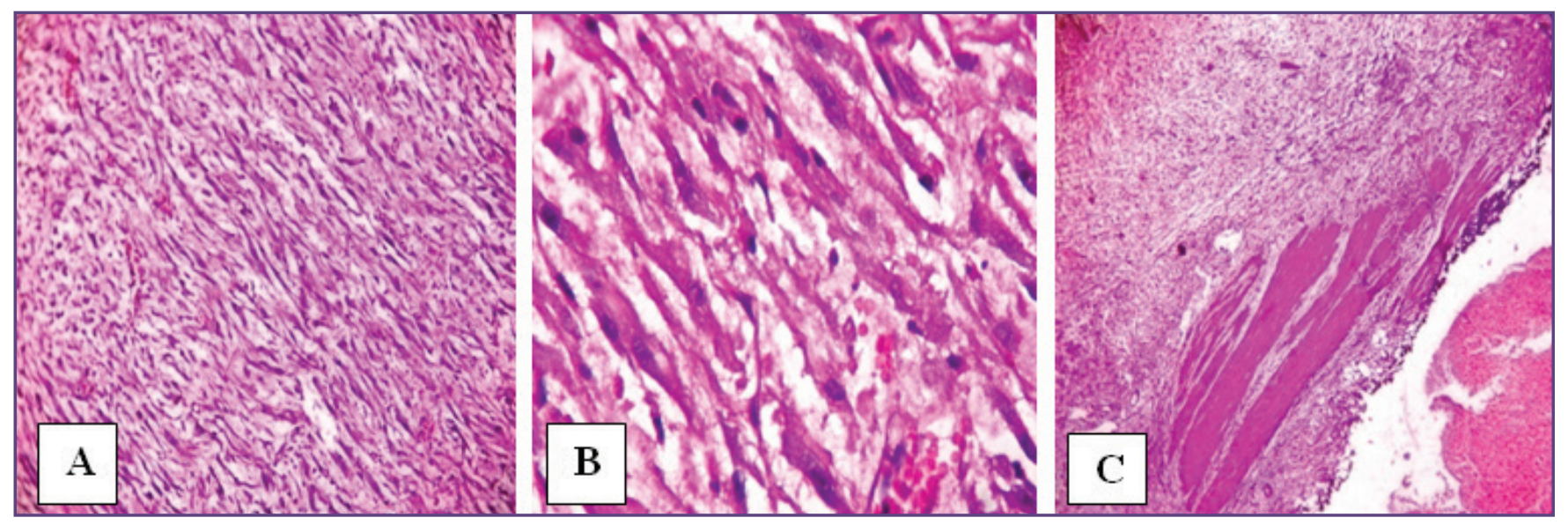

Fig. 1: Fascicles of spindle cells (A) with nuclear atypia (B) and involvement of muscular layer (C).
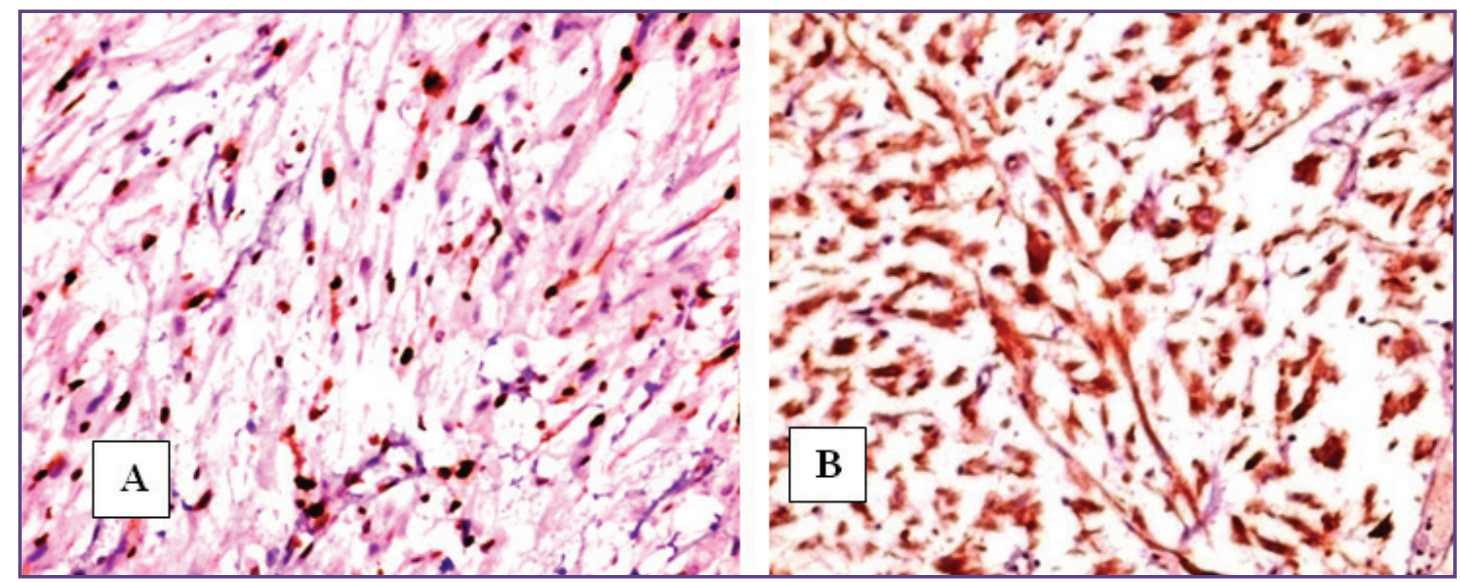

Fig. 2: Immunohistochemistry showing high Ki67 (A) and strong positivity for ALK-1.

\section{Discussion}

Inflammatory myofibroblastic tumor (IMT) of the urinary bladder is an unusual spindle cell lesion that exhibits cytologic atypia, infiltrative growth, and mitotic activity mimicking malignant tumors, such as leiomyosarcoma, rhabdomyosarcoma, and sarcomatoid carcinoma ${ }^{[4]}$. It is idiopathic and no known predisposing condition exist for myofibroblastic tumor of the bladder ${ }^{[5]}$. However, trauma is considered as a strong possible cause in many cases. Essential criterion for the diagnosis of IMT are : spindle myoepithelial cell proliferation and lymphocyte infiltrate ${ }^{[7]}$.
Studies have demonstrated clonality, suggesting that they are neoplastic rather than reactive in nature; a possible exception is those that occur immediately following instrumentation $^{[8]}$ (post-operative spindle cell nodule). Post-operative spindle cell nodule (PSCN) is a rare nonneoplastic lesion of the bladder consisting of a reactive proliferation of spindle cells, occurring between several weeks or months following surgical intervention, such as transurethral resection or biopsy ${ }^{[9]}$. Recently, anaplastic lymphoma kinase (ALK) gene translocation or ALK protein expression in IMT has been reported, in relatively 
younger age group. The detection of ALK protein and ALK gene rearrangements are useful in distinguishing IMT from other spindle cell malignancies in the urinary bladder ${ }^{[4,10]}$. Coffin $\mathrm{CM}$ et al ${ }^{[3]}$ concluded from his study that ALK reactivity was associated with local recurrences, but not distant metastasis, which was confined to ALKnegative lesions.

The case under discussion is an aggressive spindle cell tumor on account of its invasion of muscular layer of the bladder and involvement of the base of the tumor, coupled with an atypical cellular morphology which also showed high proliferative index with Ki67 on immunohistochemistry. Similar aggressive histological features were also identified by case studies of Etani $\mathrm{T}$ et $a l^{[1]}$, Yagnik V et $a l^{[7]}$ and Tanny SPT et al ${ }^{[11]}$. However, benign nature of the tumor was also considered by the histological features discussed above.

The immunostains done for the tumor in our case also assisted in clinching the final diagnosis. A strong positivity for ALK with a high proliferative index (Ki 67), and focal positivity for SMA, projected the tumor as an atypical form of IMT. Positivity for SMA and ALK-1 was also seen in the case report of Yagnik V et al ${ }^{[7]}$. However, desmin was additionally expressed in that case study. The case report of Etani $\mathrm{T}$ et al ${ }^{[1]}$ showed positivity for ALK, vimentin and SMA, but was negative for desmin. Study of the immunostains also ruled out the malignant sarcomatous proliferations, as discussed above.

It is important to rule out IMT and PSCN from malignant spindle cell proliferations of the bladder, in order to avoid radical surgical interventions. IMTs and PSCNs can be treated with total excision alone, followed by a close surveillance.

\section{Conclusion}

IMT needs to be suspected in case of spindle cell proliferation with/without atypia and chronic inflammatory infiltrate in urinary bladder, particularly with no history of instrumentation (which rules out PSCN). A thorough histopathological examination along with a suitable immunohistochemistry panel is needed for a final diagnosis. Identification of spindle cell proliferative lesions like IMT is important to avoid radical resections.

\section{References}

1. Etani T, Naiki T, Nagai T, Lida K, Ando R, Naiki-Ito A et al. Inflammatory Myofibroblastic tumor of urinary bladder; A case report. Case Rep Oncol 2016; 9(2) : 464-469

2. Fletcher CDM, Bridge JA, Hogendoorn P, Mertens F. WHO Classification of Tumours of Soft Tissue and Bone. Vol. 5. Lyon : IARC Press ; $2013 ; 83-84$.

3. Coffin CM, Hornick JL, Flethcher CD. Inflammatory myofibroblastic tumor: Comparison of clinicopathologic, histologic, and immunohistochemical features including ALK expression in atypicall and aggressive cases. Am J Sur Pathol 2007; 31(4): 509-520

4. Rao RN, Ranjan P, Singla N, Pandey R. Inflammatory myofibroblastic tumor of the urinary bladder diagnosed by anaplastic lymphoma kinase immunostaining. Urol Ann 2012; 4(2): 115-118

5. Dobrosz Z, Rys J, Palen P, Wlaszczuk P, Ciepiela M. Inflammatory myofibroblastic tumor of the bladder - an unexpected case co-existing with an ovarian teratoma. Diagnost Pathol 2014:9;138

6. Pettinano G, Manivel JL, De Rosa N, Dehner LP. IMT (plasma cell granuloma): Clinico-pathologic study of 20 cases with immunohistochemical and ultrastructural observations. Am J Clin Pathol 1990; 94: 538-546

7. Yagnik V, Chadha A, Chaudhari S, Patel K. Inflammatory myofibroblastic tumor of the urinary bladder. Urol Ann 2010; 2(2): 78-79

8. Mills SE. Editor. Sternberg's Diagnostic Surgical Pathology. 5th ed.; Lippincott Williams \& Wilkins; 2010

9. Zhao J, Ping H, Xing N. Post-operative spindle cell nodule of the bladder : a case report and review of literature. Oncol Lett. 2014; 7(5): 1507-1510

10. Toyonori T, Christina M, Jonathan E. ALK-1 Expression in Inflammatory Myofibroblastic Tumor of the Urinary bladder. Am J Sur Pathol 2004; 28(12): 1609-1614

11. Tanny SPT, Wang LL, Liddell HA, Longano A, Appu $\mathrm{S}$, Shahbaz S. Inflammatory myofibroblastic tumor of the urinary bladder : a case report. Urol Case Rep 2016 ; 6: 58-59

*Corresponding author:

Dr. Abhijit Kalita, House no 66, Happyvilla, Ujanbazar, Guwahati, Assam, India. Pin: 781003.

Email: abhighy1985@gmail.com

Date of Submission : 01.11.2016

Date of Acceptance : 01.02.2017

Financial or other Competing Interests: None.
Date of Publication : 28.03.2017 